\title{
La recherche sur les services de santé: pourquoi? - parce que!
}

\author{
Willy Oggiera, Christoph Bosshard \\ a Dr oec. HSG, Gesundheitsökonomische Beratungen AG, Küsnacht \\ ${ }^{b}$ Dr méd., vice-président et responsable du département Données, démographie et qualité, FMH, Berne
}

Les évolutions démographiques et économiques, et l'arrivée de nouvelles stratégies de traitement exposent le système de santé suisse à un processus de réforme permanent. Il faut développer et mettre en œuvre de nouveaux concepts de prise en charge médicale et en vérifier les bénéfices pour les patients. C'est pourquoi une recherche sur les services de santé durable et indépendante est indispensable. Il est par ailleurs primordial que cette recherche se penche sur la question des soins aux catégories de la population particulièrement importantes du point de vue de la santé publique.

Il y a quelques jours, la directrice du plus grand groupe d'assurance-maladie en Suisse a cru bon de remettre en question l'assurance-maladie sociale en réclamant des franchises de 5000 à 10000 francs. Avec cette revendication basée uniquement sur la redistribution, elle semble ignorer qu'un système d'assurance social repose sur d'autres valeurs fondamentales et, surtout, que des sommets sont déjà atteints en termes de paiements à la charge du patient.

En plus de la solidarité, le principe de confiance est ici essentiel. La relation médecin-patient est fondée sur ce principe et, en fin de compte, aussi la relation assuré-assureur.

La relation de confiance s'imprègne des règles du jeu au sein du système, mais surtout des effets de ce système. Il est donc fondamental pour une assurance sociale que les prestations promises soient bien à disposition en cas de besoin et qu'elles soient fournies dans la qualité requise, de manière appropriée et économique. C'est par exemple aussi l'exigence de l'art. 32 al. 1 LAMal. Des dispositions similaires existent aussi dans d'autres domaines de l'assurance sociale et à l'étranger.

La stratégie Santé2020 du Conseil fédéral a aussi essayé d'en tenir compte. Les références répétées à la stratégie 2020 ont sans aucun doute permis de sensibiliser (de manière accrue) à certaines priorités du Conseil fédéral. On peut dire que quelques-uns des signaux émis ont incité l'un ou l'autre acteur de la politique de santé à faire quelques pas en vue d'anticiper la stratégie Santé2020.
Mais, avec quelques années de recul, les points faibles de la stratégie se font davantage remarquer.

- De nombreux objectifs sont peu opérationalisables et donc peu tangibles pour l'ensemble de la population.

- La vue d'ensemble du système se focalise plutôt sur les acteurs de la politique de santé que sur les groupes cibles importants en matière de santé publique.

- La concurrence en tant qu'instrument (et non d'idéologie) pour favoriser l'innovation au niveau des produits, de l'organisation et des processus a été (presque) entièrement négligée.

- Bien que la stratégie Santé2020 mentionne que l'état de santé en Suisse est déterminé à $60 \%$ par des facteurs environnementaux et comportementaux extérieurs à la politique de santé (éducation, sécurité sociale, situation de travail, revenu, environnement, transports, situation de l'habitat), les objectifs qu'elle formule se limitent aux $40 \%$ de facteurs relevant de la politique de santé.

Pour répondre au principe de confiance évoqué plus haut, il est primordial que la recherche sur les services de santé se penche sur la question des soins aux groupes cibles particulièrement importants du point de vue de la santé publique. En effet, lorsque ceux-ci bénéficient d'une bonne prise en charge médicale, on peut légitimement penser que les soins à l'ensemble de la population sont également bons.

Rappelons quels groupes cibles sont particulièrement importants du point de vue de la santé publique: les 
1 «Das Gesundheitssystem der USA ist Murks» (le système de santé des USA est bâclé), interview avec le Prof. Dr Uwe Reinhardt, économiste de la santé à l'université de Princeton, USA, dans: Deutsches Ärzteblatt. 24. octobre 2014;111(43):1844-5. personnes âgées, les personnes socialement défavorisées et les patients bénéficiant de soins coûteux.

Il va de soi que des recoupements sont possibles entre ces groupes, mais, au nom de la transparence et pour améliorer les bases de décision, il est préférable de les analyser un à un dans un premier temps, et de se pencher ensuite sur leurs interactions.

Un système qui - comme l'a formulé l'économiste de la santé Uwe E. Reinhardt en s'appuyant sur l'exemple des USA - est le meilleur du monde si l'on est intelligent et en bonne santé («Mais si l'on n'est ni intelligent ni en bonne santé, c'est dur ici.»), n'est pas pertinent en termes de politique de soins. ${ }^{1}$

La recherche sur les services de santé doit donc se focaliser sur les groupes cibles les plus importants, ceux pour lesquels il existe un besoin d'agir, ceux sur lesquels le marché n'a pas d'effet et dans lesquels il est possible de faire beaucoup pour améliorer la qualité de vie. Dans ce cas, l'action de l'Etat est justifiée.

Il convient de privilégier la concurrence là où elle est un instrument qui fonctionne dans les limites de conditions cadres claires.

Aujourd'hui - précisément en Suisse -, la recherche sur les services de santé continue cependant de s'axer sur de nombreux aspects encouragés par l'Etat, mais secondaires pour la prise en charge médicale. Cela doit changer. La recherche dans ce domaine ne remplit sa fonction que si elle apporte un plus, comme le sel dans la soupe. Si les principaux acteurs de la santé, les médecins, les thérapeutes, le personnel infirmier et les autres fournisseurs de prestations, ne relèvent pas ce défi, ils ne faut pas qu'ils s'étonnent lorsque l'Etat intervient. Mais au moins, ils sont en droit de se poser la question de savoir si le résultat sera meilleur.

Le prix zurichois de la recherche sur les services de santé de la direction de la santé du canton de Zurich sera décerné pour la première fois à l'occasion du $5^{\mathrm{e}}$ forum éponyme du $5 / 6$ juillet 2018 à Zurich. La manifestation organisée par la société MediCongress $\mathrm{GmbH}$ a été soutenue dès le début par la direction de la santé du canton de Zurich et son directeur, le conseiller d'Etat, le DrThomas Heiniger, et par la FMH. Cette année, le forum traitera de la prise en charge médicale des personnes âgées, des personnes socialement défavorisées et des patients bénéficiant de soins coûteux. Programme et inscription sur www.medicongress.ch 